\title{
Observational study of the use of serial Non-EPI DWI MRI scans to determine the growth of cholesteatoma
}

\author{
Wendy Smith ${ }^{1}$ and Leena Naidu ${ }^{2}$ \\ ${ }^{1}$ Leeds Teaching Hospitals NHS Trust \\ ${ }^{2}$ Kettering General Hospital NHS Foundation Trust
}

July 7, 2020

\begin{abstract}
Introduction: It is an established practice to use non-EPI DWI MRI scans to detect the presence of cholesteatoma post operatively. In the present era of Covid-19 where routine surgery to remove cholesteatoma has been suspended resulting in potentially unprecedented demands on the service, a review of serial MRI scans performed over a 7 year period was undertaken to determine the rate of growth of cholesteatoma. Materials and methods: A retrospective longitudinal study identified 24 middle ear cholesteatomas in 17 patients with serial non-EPI DWI MRI scans (having excluded those having surgical intervention between scans) for a median period of 33 months (range of 6-91 months). Cholesteatomas were measured by the first author and by the consultant radiologist. Results: Of 24 cholesteatomas, 1 resolved completely, 5 reduced, 6 stayed the same size, 4 grew slowly and 8 grew significantly. Conclusion: Non-EPI DWI MRI scans to determine cholesteatoma growth in asymptomatic ears is useful in triaging patients in the Covid-19 era. Key words: Cholesteatoma MRI scans covid-19
\end{abstract}

A retrospective Longitudinal study of the use of serial Non-EPI DWI MRI scans to determine the growth of cholesteatoma

Abstract:

Introduction: It is an established practice to use non-EPI DWI MRI scans to detect the presence of cholesteatoma post operatively. In the present era of Covid-19 where routine surgery to remove cholesteatoma has been suspended resulting in potentially unprecedented demands on the service, a review of serial MRI scans performed over a 7 year period was undertaken to determine the rate of growth of cholesteatoma.

Materials and methods: A retrospective longitudinal study identified 24 middle ear cholesteatomas in 17 patients with serial non-EPI DWI MRI scans (having excluded those having surgical intervention between scans) for a median period of 33 months (range of 6-91 months). Cholesteatomas were measured by the first author and by the consultant radiologist.

Results: Of 24 cholesteatomas, 1 resolved completely, 5 reduced, 6 stayed the same size, 4 grew slowly and 8 grew significantly.

Conclusion: Non-EPI DWI MRI scans to determine cholesteatoma growth in asymptomatic ears is useful in triaging patients in the Covid-19 era.

Key words: Cholesteatoma MRI scans covid-19

Key Points:

- Non-EPI DWI MRI scans are useful to monitor the growth behaviour of cholesteatoma in asymptomatic ears. 
- Only half of the 24 cholesteatomas in this study demonstrated any increase in size over 6 to 91 months (median 33months).

- This investigation could be invaluable in managing patients conservatively even when cholesteatoma is found on post-operative scans.

- Cholesteatomas in adults with bilateral disease and cholesteatomas in children appear to be more "aggressive", being more likely to recur after surgery and may require multiple operations to clear the disease.

- Monitoring cholesteatoma growth by serial MRI scans should be considered in patients where surgery is to be avoided either in patients with co-morbidities or in managing patients in the Covid-19 era.

Text:

The use of serial Non-EPI DWI MRI scans to determine the growth of cholesteatoma

Introduction: Middle ear cholesteatoma is mainly diagnosed clinically and the gold standard treatment is surgical removal of the disease. High-resolution computed tomography (HRCT) scan of the temporal bone provides useful information on the anatomy and location of potential disease but it is unable to differentiate cholesteatoma from cholesterol granuloma, fluid or other non-cholesteatoma soft tissue ${ }^{1}$. The use of the canal wall-up approach to the surgical management of cholesteatoma or cartilage and hydroxyapatite granules to obliterate cavities prevents the detection of residual cholesteatoma clinically following surgery. It is now an established practice to use non-EPI DWI MRI scans to detect the presence of cholesteatoma post operatively with studies even reporting sensitivity and specificity of up to $100 \%^{2-6}$.

In the present era of covid-19 where routine surgery to remove cholesteatoma has been suspended resulting in potentially unprecedented demands on the service, a review of serial MRI scans performed over a 7 year period was conducted to identify the behaviour with specific reference to the rate of growth rate of middle ear cholesteatoma. This was to determine whether all cholesteatoma requires surgical removal and secondly a means to prioritise cases for surgery particularly during a pandemic crisis.

Materials and methods: A retrospective longitudinal study of patients under the care of the first author who had had serial non-EPI DWI MRI scans between 2015 and 2019 were identified. The cholesteatomas in patients who had had surgical intervention on the ear between the serial scans were excluded. 20 cholesteatomas were detected on post-operative scans; 4 cases occurred in non-operated ears. Some patients who had cholesteatoma detected in otherwise asymptomatic ears elected to be managed conservatively with serial MRI scanning. They declined surgery due to the potential risks of surgery or personal circumstances whilst 2 were deemed medically unfit for surgery.

There was a total of 24 cholesteatomas in 23 ears in 17 patients. There were 10 males, 7 females. The age range was 10-72 years ( 3 were children) with median age of 32 years. All ears were examined under the microscope and an attic pocket was found in 6; 4 representing recurrent disease but in 2 this was new disease in a non-operated ear. The remaining 18 cholesteatomas were found in asymptomatic ears with no obvious cholesteatoma. The median period for monitoring was 33 months with a range of 6-91 months. All had at least 2 serial non-EPI DWI MRI scans.

The MRI scans were evaluated independently by an experienced head and neck consultant radiologist and the consultant otologist. Cholesteatoma was diagnosed in the presence of restricted diffusion on non-EPI DWI sequence and a low signal intensity on ADC mapping. Both of these sequences as well as the T1 and T2 were reviewed since DWI artefacts can occur (due to so-called T2 shine through, wax, cholesterol granuloma). The size of the cholesteatomas was independently measured as per the usual practice by the otologist (maximal dimension in $\mathrm{mm}$, using images taken in the coronal plane) and by the radiologist (largest axial dimension in $\mathrm{mm}$ ) to predict the growth behaviour of the cholesteatomas.

Results: Table 1 shows the serial measurements (in $\mathrm{mm}$ ) of the largest diameter of the cholesteatomas in the coronal and axial planes with the interval between the scans in months. Figure 1 demonstrates that there appears to be very little correlation $(\mathrm{R} 2=0.0716)$ between the growth behaviour of cholesteatomas 
based on the maximum dimensions obtained for each cholesteatoma when measured in the axial and coronal planes which is also seen in Figure 2. Despite this, 7 of 24 cholesteatomas were assessed as behaving in the same manner by the radiologist and otologist and in 9 of 24 cholesteatomas to within 1 group (defined by an increase $>4 \mathrm{~mm}$, a small increase of $>1 \mathrm{~mm}$ but $<4 \mathrm{~mm}$, the same $-1 \mathrm{~mm}$ to $+1 \mathrm{~mm}$, a small decrease $>-1 \mathrm{~mm}$ to $<-4 \mathrm{~mm}$, a decrease $>4 \mathrm{~mm}$, resolved) of each other. The overall growth in the coronal plane was greater than in the axial in two-thirds of the cholesteatomas but the average difference in the two measurements was $0.9 \mathrm{~mm}$ with measurements in the coronal plane tending to be larger.

Of the 24 cholesteatomas 1 resolved completely, 5 reduced, 6 stayed the same size whilst 4 grew slowly and 8 grew significantly.

Discussion:

It has been advocated that cholesteatoma should be managed by surgical removal in a timely fashion to prevent the complications of the disease which include hearing loss (both conductive and sensorineural), facial nerve palsy, erosion of vestibular apparatus as well as infection that may spread intracranially.

This is the first study, to our knowledge, that has monitored the growth behaviour of both operated and non-operated middle ear cholesteatomas using non-EPI DWI MRI scans. The study demonstrated some consistency between the consultant head and neck radiologist and otologist in predicting the behaviour of cholesteatoma growth although there were some inconsistencies. Review of any previous MRI scans can be useful to confirm whether an area suspicious of restricted diffusion of the non-EPI DWI sequence has developed a more obvious restricted diffusion and has increased in size as seen in images 1a and 1b. In addition to the degree of inter-variability between the otologist and radiologist, the otologist measured the largest diameter in the coronal plane whereas the radiologist measured in the axial plane. Wong et al in 2016 found that cholesteatoma expands most rapidly in the craniocaudal plane ${ }^{7}$. Measuring the greatest dimension of the cholesteatoma in the coronal plane it is therefore more likely to detect any change in the size of the disease.

20 cholesteatomas were found in operated ears. 4 were in ears that had an attic pocket and represented recurrent disease however 16 were in asymptomatic ears with no obvious cholesteatoma present clinically behind an intact tympanic membrane. Our study has found that 8 had grown in one measurement by $>4 \mathrm{~mm}$ and 4 between $1-4 \mathrm{~mm}$. 10 proceeded to have surgery however 2 were in patients deemed unfit for surgery and continue to be managed conservatively with further MRI scans. 6 cholesteatomas have remained unchanged in size, 5 have decreased by $1-4 \mathrm{~mm}$ and one cholesteatoma had resolved. Even prior to the introduction of non-EPI DWI MRI scans, Gristwood and Venables in 1976 estimated the growth rate of cholesteatoma by measuring the volume of cholesteatoma found at second look surgery and dividing it by the days between initial and second look surgery ${ }^{8}$. They found an exponential growth pattern, not found by Hellingmann et al in $2019^{9}$. The latter followed patients up to 4.5 years and found variation in the growth rate of different cholesteatomas even in the same patient. Hellingman et al found a large individual variation in the growth rate of residual cholesteatoma in 10 patients after subtotal petrosectomy with a rho value of 0.32 for the correlation between growth rate and volume of cholesteatoma at detection ${ }^{9}$. They concluded that where the volume of cholesteatoma is small with room to grow before destroying any remaining structures, a wait and scan policy could be considered. This suggests that serial MRI scans may be a useful adjunct to determine when and what priority should be given to patients listed for surgery to remove cholesteatoma

The rate of growth of these 24 cholesteatomas per year was then calculated and found to vary from $-0.2 \mathrm{~mm}$ to $19.9 \mathrm{~mm} /$ year with a median growth of $0.6 \mathrm{~mm} /$ year. This is important to consider when advocating the timing and frequency of follow-up scans. It has been our practice to arrange non-EPI DWI MRI scans in patients around 12 months and 5 years post-surgery with additional scans at 3 years in children, where there was a suspected or a small cholesteatoma detected on a scan or if clinically indicated. Our study first detected cholesteatoma in this cohort of patients at 10 to 27 months (median of 14.5 months) post-surgery. Wong et al suggested that in patients where routine second look surgery is not performed following canal wall up surgery, a follow-up scan at 1-2 years is likely to detect most residual disease ${ }^{7}$. Pai et al in 2019 also 
found the growth rate of residual cholesteatoma to be highly variable, ranging from static over three years to an estimated value of $29 \mathrm{~mm} /$ year $^{10}$. They therefore recommended interval imaging for a minimum of 5 years in stable ears following definitive cholesteatoma surgery with additional interval scan between 2 and 3 years postoperatively if indicated ${ }^{10}$.

4 of the cholesteatomas in this study occurred in non-operated ears and in only 2 of these was (an attic) cholesteatoma clinically present. The other patients were asymptomatic and were incidental findings on MRI scans performed to follow-up previous disease in the other ear. Of these primary cholesteatomas 2 showed significant growth, one minimal growth and one actually regressed. Wong et al in 2016 reported the progress of 12 cases of non-operated middle ear cholesteatoma using non EPI DWI MRI imaging describing only one case of rapid progression, a third had a mean growth of 11.9\%/year; 7 showed evidence of a mean regression of 53\%/year with 3 having resolved completely over a 17 month period $^{7}$. Although the study had some limitations since it reported on a small cohort of patients who did not have surgery to confirm the "true" extent of disease, it does raise the issue as to if and when cholesteatomas even in non-operated ears require to be removed surgically.

Review of these 24 cases suggests that significant growth $(>4 \mathrm{~mm})$ is mostly likely in cholesteatoma found in children or in adults with bilateral disease. In fact, in all 8 patients with $>4 \mathrm{~mm}$ increase in the size of their cholesteatoma, all had bilateral disease, two of whom were diagnosed in childhood. A number of theories have been suggested as to the variation in the growth rates seen including the effect of the host innate immune response and genomic alterations found in cholesteatoma ${ }^{9-11}$.

In addition to confirming the presence of residual cholesteatoma in asymptomatic post-operative ears, this study has demonstrated the value of serial non-EPI DWI MRI scans in providing information on the growth behaviour of cholesteatoma. Interestingly only half of the 24 cholesteatomas actually increased in size with 6 remaining static, the rest reducing in size with one resolving completely. This may suggest that we may in fact be over-treating some cholesteatomas and serial scanning may offer an appropriate alternative to surgical removal especially in patients with co-morbidities or where surgery is limited due to the impact of Covid-19 or any future pandemics.

References:

1. Tierney PA, Pracy P, Blaney SP, Bowdler DA. An assessment of the value of the preoperative computed tomography scans prior to otoendoscopic 'second look' in intact canal wall mastoid surgery. Clin Otolaryngol Allied Sci. 1999;24(4):274-276 doi:10.1046/j.1365-2273.1

2. Garrido L, Cenjor C, Montoya J, Alonso A, Granell J, Gutiérrez-Fonseca R. Diagnostic capacity of non-echo planar diffusion-weighted MRI in the detection of primary and recurrent cholesteatoma. Acta Otorrinolaringol Esp. 2015;66(4):199-204 doi:10.1016/j.otorri.2014.07.006

3. Aarts MC, Rovers MM, van der Veen EL, Schilder AG, van der Heijden GJ, Grolman W. The diagnostic value of diffusion-weighted magnetic resonance imaging in detecting a residual cholesteatoma. 2010;143(1):12-16. doi:10.1016/j.otohns.2010.03.023 e

4. Li PM, Linos E, Gurgel RK, Fischbein NJ, Blevins NH. Evaluating the utility of non-echo-planar diffusion-weighted imaging in the preoperative evaluation of cholesteatoma: A meta-analysis. Laryngoscope 2013;123(5):1247-1250

5. De Foer B, Vercruysse JP, Bernaerts A, et al. Detection of postoperative residual cholesteatoma with non-echo-planar diffusion-weighted magnetic resonance imaging. Otol Neurotol. 2008;29(4):513-517 doi:10.1097/MAO.0b013e31816c7c3b

6. van Egmond SL, Stegeman I, Grolman W, Aarts MC. A Systematic Review of Non-Echo Planar Diffusion-Weighted Magnetic Resonance Imaging for Detection of Primary and Postoperative Cholesteatoma. Otolaryngol Head Neck Surg. 2016;154(2):233-240 999.00238.x

7. Wong PY, Lingam RK, Pal S et al. Monitoring progression of 12 cases of non-operated middle ear cholesteatoma with non-echoplanar diffusion weighted magnetic resonance imaging : Our experience. Otology \& Neurotology 37:1573-1576 2016, Otology \& Neurotology, Inc.

8. Gristwood RE, Venables WN. Growth rate and recurrence of residual epidermoid cholesteatoma after 
tympanoplasty. Clin Otolaryngol. 1976;1:169-182

9. HellingmanA.C, Logher JLE, Kammeijer Q,Waterval JJ, Ebbens FA, van Spronsen E. Measuring growth of residual cholesteatoma in subtotal petrosectomy, Acta Oto-Laryngologica, 2019;139:5,415420 DOI: $10.1080 / 00016489.2019 .1578413$

10. Pai I, Crossley E, Lancer H, Dudau C, Connor S Growth and Late Detection of Post-Operative Cholesteatoma on Long Term Follow-Up With Diffusion Weighted Magnetic Resonance Imaging (DWI MRI). Otology \& Neurotology. 2019;40(5):638-644 doi: 10.1097/MAO.0000000000002188

11. Kuo CL, Shiao AS, Yung M, et al. Updates and knowledge gaps in cholesteatoma research. Biomed Res IntEpub. 2015;2015:1.

\section{Hosted file}

Table 1 clin otol mri growth chole.docx available at https://authorea.com/users/339886/ articles/466752-observational-study-of-the-use-of-serial-non-epi-dwi-mri-scans-todetermine-the-growth-of-cholesteatoma

\section{Hosted file}

clin otol fig $1 \mathrm{mri}$ growth chole.docx available at https://authorea.com/users/339886/ articles/466752-observational-study-of-the-use-of-serial-non-epi-dwi-mri-scans-todetermine-the-growth-of-cholesteatoma

\section{Hosted file}

clin otol fig $2 \mathrm{mri}$ growth chole.docx available at https://authorea.com/users/339886/ articles/466752-observational-study-of-the-use-of-serial-non-epi-dwi-mri-scans-todetermine-the-growth-of-cholesteatoma

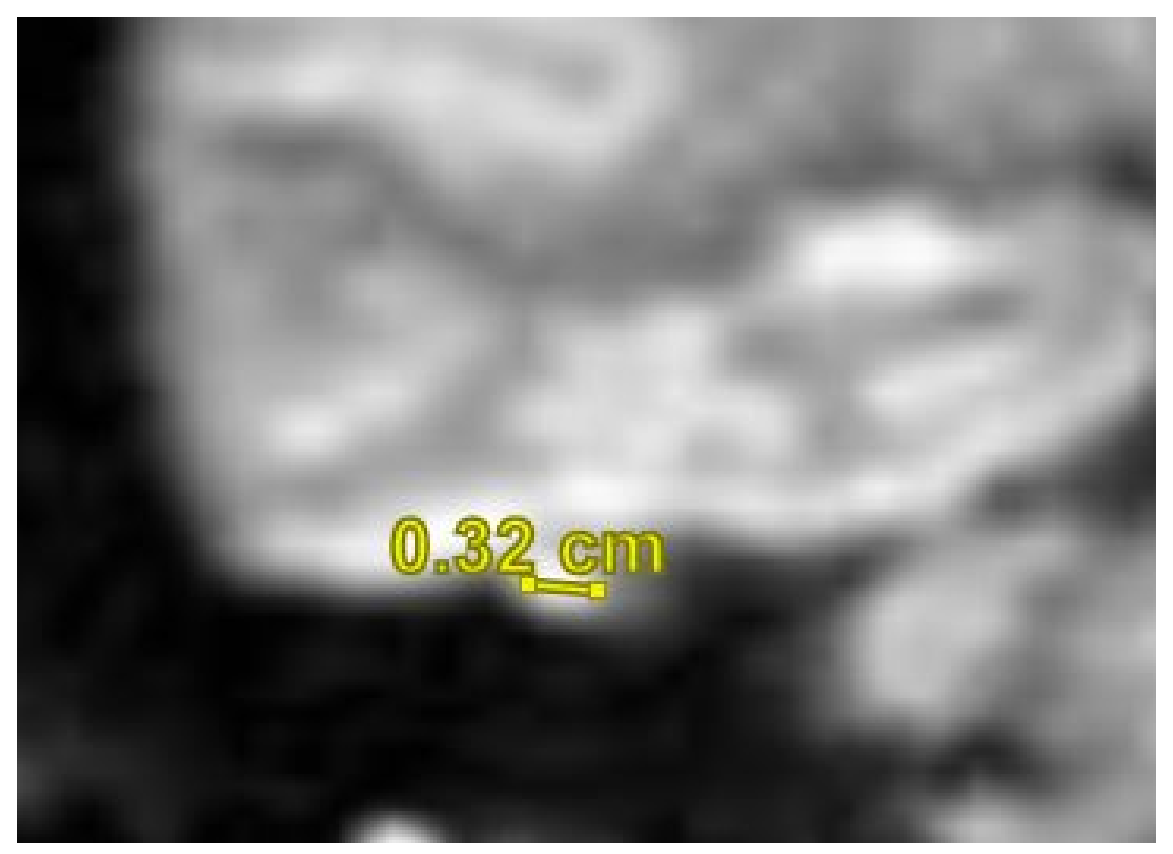


(0.92. $\mathrm{cm}$ 